\section{NOVA TELLVS}

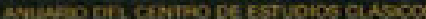

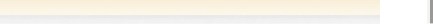

Nova Tellus

ISSN: 0185-3058

novatelu@servidor.unam.mx

Centro de Estudios Clásicos

México

Vargas Valencia, Aurelia

Aspectos lingüísticos de índole sistemática en las Instituciones de Justiniano

Nova Tellus, vol. 27, núm. 2, 2009, pp. 181-202

Centro de Estudios Clásicos

Distrito Federal, México

Disponible en: http://www.redalyc.org/articulo.oa?id=59115485007

Cómo citar el artículo

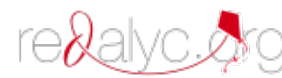

- Número completo

- Más información del artículo

Página de la revista en redalyc.org

Sistema de Información Científica

Red de Revistas Científicas de América Latina, el Caribe, España y Portugal

Proyecto académico sin fines de lucro, desarrollado bajo la iniciativa de acceso abierto 


\title{
Aspectos linguiísticos de índole sistemática en las Instituciones de Justiniano
}

\author{
Aurelia VARGAS VALENCIA \\ Universidad Nacional Autónoma de México \\ aurelia@servidor.unam.mx
}

\begin{abstract}
RESUMEN: Este artículo pone en evidencia aspectos que tienden a la sistematicidad en las Instituciones de Justiniano, primera parte del Corpus Iuris Civilis, una obra que ha tenido gran trascendencia en la historia jurídica de Occidente. Se muestra la coherencia en planos del texto como los que se refieren a la temática y al estilo, pero sobre todo a elementos lingüísticos - explicaciones etimológicas principalmente - que, por su frecuencia, dan al texto un carácter acorde con sus propósitos. Éstos fueron, como es sabido, la docencia del Derecho y los principios de ley vigentes en tiempos del emperador Justiniano (s. vI), quien a su vez actúa como recuperador de la jurisprudencia romana clásica y de la legislación imperial que le precedieron.
\end{abstract}

$$
* * *
$$

ABSTRACT: This study brings to light some aspects of Justinian's Institutions - which are the first part of the Corpus Iuris Civilis, a work that has had a transcendent role in the history of Western jurisprudence- in which a trend to a systematical treatment can be seen. The conformity of the text with its purposes is showed by pointing out the frequent use of several resources ranging from thematic and stylistic coherence between different text planes to, more importantly, linguistic devices such as etymological explanations. As is widely known, among the aims pursued by this law compilation were teaching of Law and upholding of the legal principles prevailing at the time of emperor Justinian (vI century a. D.), who thus acted as a retriever of Classical Roman Jurisprudence and of imperial legal codes that existed before him.

PAlabras Clave: Justiniano, Corpus Iuris Civilis, Institutiones, estructura, estilo, lengua, objetivos, docencia y legislación.

KeYwords: Justinian, Corpus Iuris Civilis, Institutiones, language, structure, style, teaching and legislation.

RECEPCIÓN: 3 de febrero de 2009.

ACEPTACIÓN: 11 de mayo de 2009. 


\title{
Aspectos linguiísticos de índole sistemática en las Instituciones de Justiniano ${ }^{1}$
}

\author{
Aurelia VARgas VALENCIA
}

\section{Preámbulo}

El propósito del este trabajo es evidenciar algunos aspectos estructurales, principalmente lingüísticos, de las Instituciones de Justiniano, obra de índole legal promulgada en el año $533 \mathrm{~d}$. C., y utilizada también para la docencia. Fue elaborada en Constantinopla a partir de fuentes anteriores de la legislación y la jurisprudencia romanas, mismas que habían sido aprovechadas para la composición del Corpus Iuris Civilis del cual forma parte, además del Código, cuya primera edición fue promulgada en 528 d. C., y del Digesto, cuando sus trabajos estaban por concluirse. ${ }^{2} \mathrm{El}$ tratar los aspectos estructurales de las Instituciones implica, en buena medida, dilucidar aquellos elementos que llegan a ser sistemáticos en la obra, aunque no necesariamente en los términos rígidos que expresa la definición de "sistema" en el Diccionario de la Academia, a saber, "conjunto de reglas o principios sobre una materia racionalmente enlazados entre sî", ${ }^{3}$ sino más bien como aspectos que, por su recurrencia a lo largo del texto, llegan a tener casi esa categoría.

\footnotetext{
${ }^{1}$ Una versión de este texto fue presentada como conferencia magistral en el XVI Congreso Latinoamericano de Derecho Romano, en San José de Costa Rica, el 21 de julio de 2008.

2 Vid. Álvaro D’Ors, Derecho Privado Romano, § 66, n. 1.

${ }^{3}$ Real Academia Española, Diccionario de la lengua española, s. v. "sistema".
} 


\section{Las Instituciones de Justiniano entre otras Instituciones}

Por mandato del emperador, la composición de las Institucio$n e s^{4}$ fue encomendada a una comisión de juristas constituida por Teófilo, Doroteo y Triboniano. ${ }^{5}$ Éste presidió y supervisó el trabajo que seguía una tradición en la elaboración de instituciones, la cual, según Ortolán, ${ }^{6}$ inició con las Instituciones de Gayo y concluyó precisamente con las de Justiniano, aseveración que, a mi juicio, habría que contrastar con una tradición más antigua que nos remite a obras de esta índole en otras áreas del conocimiento, como, por ejemplo, las Instituciones oratorias de Marco Fabio Quintiliano, escritas en el siglo primero de nuestra era.

Además de Justiniano y Gayo, autores de Instituciones fueron también Marciano, Florentino, Paulo, Ulpiano y Calístrato. Las Instituciones de todos ellos, pero en especial las de Gayo, junto con sus Res cottidianae, sirvieron de base para la redacción de las de Justiniano, además de varias constituciones imperiales, como él mismo lo refiere en el parágrafo 6 de la constitución Imperatoriam:

Las cuales, compuestas a partir de todas las instituciones antiguas, pero sobre todo de los comentarios de nuestro Gayo, tanto de sus Instituciones como de sus Res cottidianae, y de los comentarios de muchos otros... ${ }^{7}$

\footnotetext{
${ }^{4}$ En relación con los vocablos con que suele denominarse esta obra de Justiniano -Instituta o Institutiones-, véase A. Vargas, Las Instituciones de Justiniano en la Nueva España, p. 28, n. 38.

${ }^{5}$ Cumque hoc deo propitio peractum est, Triboniano viro magnifico magistro et exquaestore sacri palatii nostri nec non Theophilio et Dorotheo viris illustribus antecessoribus, quorum omnium sollertiam et legum scientiam et circa nostras iussiones fidem iam ex multis rerum argumentis accepimus, convocatis specialiter mandavimus, ut nostra auctoritate nostrisque suasionibus componant institutiones ... (Inst., Proemium, § 3).

${ }^{6}$ M. Ortolán, Instituciones de Justiniano, trad. esp. Francisco Pérez de Anaya y Melquiades Pérez Rivas, p. 18.

${ }^{7}$ Quas ex omnibus antiquorum institutionibus et praecipue ex commentariis
} 
De todos estos textos de instituciones, fueron las de Justiniano las que tuvieron gran influencia en los siglos posteriores, tanto en la enseñanza del Derecho en universidades europeas y del Nuevo Mundo, como en su utilización en calidad de fuente legislativa.

\section{Las Instituciones, un texto con carácter de ley vigente}

Desde su elaboración, las Instituciones de Justiniano fueron concebidas como un texto con carácter de ley vigente. El emperador lo manifiesta en el mismo parágrafo 6 de la constitución Imperatoriam, con las siguientes palabras: "las ... hemos leído y conocido, y les hemos dado el plenísimo vigor de nuestras constituciones". 8

En distintos pasajes de ese texto, Justiniano deja ver el interés que tenía por publicar esta obra, pero sobre todo enfatiza (§ 2 de la misma constitución) la ardua labor que implicó llevarla a cabo y la forma en que se hizo:

Después que redujimos a una espléndida consonancia las sacratísimas constituciones antes confusas, hemos dirigido nuestro cuidado también a los inmensos volúmenes de la antigua jurisprudencia, y caminando como sumergidos en un abismo de dificultades, concluimos, con el favor del cielo, tan arduo trabajo. ${ }^{9}$

Fue, en efecto, un trabajo que él leyó y revisó antes de autorizar su publicación, como ya lo hemos visto en los términos "legimus et cognovimus" (Imperatoriam, 6). Este es un dato

Gaii nostri tam institutionum quam rerum cottidianarum aliisque multis commentariis compositas...

${ }^{8}$ Quas ... et legimus et cognovimus et plenissimum nostrarum constitutionum robur eis accommodavimus.

${ }^{9}$ Et cum sacratissimas constitutiones antea confusas in luculentam ereximus consonantiam, tunc nostram extendimus curam et ad immensa prudentiae veteris volumina, et opus desperatum quasi per medium profundum euntes caelesti favore iam adimplevimus. 
de no poca importancia, si se considera que representa la utilidad práctica que tuvo para su momento histórico, es decir, la recepción del derecho romano en la nueva capital del imperio, en la nueva Roma, como fue llamada aquella ciudad situada en las orillas del Bósforo, ${ }^{10}$ que había sido fundada como colonia griega en el siglo VII a. C. con el nombre de Bizancio, y refundada por Constantino bajo el nombre de Constantinopla en 330 d. C. Así, desde la Urbs presidida por Justiniano, el derecho romano volvió a extenderse - actualizado y adecuado a sus nuevas circunstancias - a todos los pueblos que conformaban el nuevo imperio que, bajo dicho emperador, alcanzó una expansión similar a la que había tenido anteriormente el imperio romano. Él mismo lo manifiesta en el título que trata sobre las fianzas (Inst., 4, 11, 7), pero que bien puede aplicarse a todos los temas incluidos en su legislación: "Que esta forma <se aplique> no sólo en nuestra regia ciudad, sino también en todas las provincias"; 11 voluntad que expresó también en su proemio: "por las leyes promulgadas o compuestas por nosotros se rigen todos los pueblos"12 (Imperatoriam, 1).

\section{Las Instituciones, un texto didáctico}

Antes de revisar los aspectos de lengua característicos de la obra, es preciso delinear el marco en el que se circunscriben.

${ }^{10}$ Bizancio era la ciudad con la más vieja tradición imperial de todo el mundo. Noventa y dos emperadores se habían sentado en su trono. Siendo Arcadio emperador de Constantinopla, en el año 395 el Imperio romano queda definitivamente dividido. Así, el Imperio Bizantino, que bajo Justiniano (527-565) alcanzó una expansión similar a la que había tenido el Imperio Romano (exceptuando Hispania y Galia), se inclinó progresivamente hacia Oriente a partir del reinado de Heraclio (610-641). De este modo, asediado por los árabes y más tarde por los búlgaros (venidos de Asia), Bizancio se convertiría durante varios siglos en el bastión de la cristiandad frente al islam.

${ }^{11}$ Quam formam non solum in hac regia urbe, sed et in omnibus nostris provinciis...

${ }^{12}$ Omnes vero populi legibus iam a nobis vel promulgatis vel compositis reguntur. 
Por principio, como se apreciará, puede decirse que el concepto "sistema" en las Instituciones de Justiniano, se manifiesta fundamentalmente en la intención didáctica. Esta característica se muestra de diversos modos, a tal punto que da forma, estructura y sentido a toda la obra.

Las instituciones habían sido un género de literatura emanado del conocimiento adquirido por los profesores de Derecho. Se caracterizaban por ser libros elementales de texto, escritos principalmente para estudiantes, ${ }^{13}$ y su inclinación didáctica se halla mencionada en la Lex Romana Visigothorum o Breviarium Alarici, promulgado en el año 506 d. C., donde se decía que el libro de texto con el que los jóvenes de esa época iniciaban sus estudios de Derecho era el de las Instituciones de Gayo; Alarico, sin embargo, presentaba en su Breviario sólo un resumen o epítome de éstas, a diferencia de Justiniano, quien introduce, como se ha señalado, sus propias disposiciones, actualizando con esto el derecho compilado, en la medida que la necesidad lo dictaba.

La intención didáctica se ve, por principio, declarada explícitamente en su propio título "Institutionum sive elementorum ... liber primus ...secundus ...”, y en el $\S 4$ de la constitución Imperatoriam donde se refiere a éstas: "para que sean los primeros elementos de toda la ciencia jurídica". ${ }^{14}$ Dichas Instituciones estaban dirigidas también, como las de Gayo, a los estudiantes de Derecho del primer año, a quienes Justiniano en el título de la Imperatoriam dedica la obra con la frase "a la juventud deseosa de <estudiar> las leyes", ${ }^{15}$ y agrega:

reciban con suma diligencia estas leyes nuestras y muéstrense de tal modo instruidos, que pueda animarlos la esperanza de

\footnotetext{
${ }^{13}$ Vid. Adolf Berger, Encyclopedic Dictionary of Roman Law, s. v. Institutiones.

${ }^{14}$ Ut sint totius legitimae scientiae prima elementa.

${ }^{15}$ Cupidae legum inventuti.
} 
llegar a gobernar el imperio en las partes que les fueren confiadas. ${ }^{16}$

Pero Justiniano no sólo declara que la obra está dirigida a los estudiantes, sino que marca él mismo la directriz que habrá de guiar la composición de las Instituciones a lo largo de sus cuatro libros. Así, desde el primer título del libro primero dice:

...empezando a exponer los derechos del pueblo romano, nos parece que pueden transmitirse más convenientemente si primero mediante una vía clara y sencilla, y luego con una diligentísima y exactísima interpretación, se transmite cada cosa. ${ }^{17}$

(Inst., 1, 1, 2)

Pues, agrega:

Si cargamos desde el principio el aún inexperto y endeble ánimo del estudiante con multitud y variedad de cosas, una de dos: o lo convertimos en desertor de sus estudios o, con gran esfuerzo de su parte, a menudo también con desconfianza - que por lo común ahuyenta a los jóvenes-, lo llevaremos más tarde a aquello que hubiera podido llegar más oportunamente, si lo hubiéramos conducido por una vía más llana, sin gran esfuerzo y sin desconfianza alguna. ${ }^{18}$

(Inst., 1, 1, 2)

\footnotetext{
${ }^{16}$ Ibidem, § 7: summa itaque ope et alacri studio has leges nostras accipite et vosmet ipsos sic eruditos ostendite, ut spes vos pulcherrima foveat toto legitimo opere perfecto posse etiam nostram rem publicam in partibus eius vobis credendis gubernare.

17 ... incipientibus nobis exponere iura populi Romani ita maxime videntur posse tradi commodissime, si primo levi ac simplici, post deinde diligentissima atque exactissima interpretatione singula tradantur.

${ }^{18}$ Alioquin si statim ab initio rudem adhuc et infirmum animum studiosi multitudine ac varietate rerum oneraverimus, duorum alterum aut desertorem studiorum efficiemus aut cum magno labore eius, saepe etiam cum diffidentia, quae plerumque iuvenes avertit, serius ad id perducamus, ad quod leniore via ductus sine magno labore et sine ulla diffidentia maturius perduci potuisset.
} 
Así, comenzar por lo sencillo sería la línea, y efectivamente esa fue la forma en que se redactó el texto, lo que podemos apreciar en varios pasajes. Por ejemplo, en el libro II (Sobre las cosas), vemos lo siguiente: después de que en Inst., 2, 1 ha explicado la clasificación de las cosas según se hallan dentro o fuera del patrimonio, en Inst., 2, 2 pr. trata "de las cosas corpóreas e incorpóreas", y habiendo explicado cada una con sus ejemplos, dice de las incorpóreas que consisten en un derecho, como la herencia, el usufructo, el uso y la habitación, las obligaciones, y las servidumbres prediales (Inst., 2, 2, 2-3). El caso es que dedica un apartado a analizar cada una de éstas (Inst., $2,3 ; 2,4 ; 2,5)$, pero deja fuera de la explicación la herencia y las obligaciones, de las cuales, cuando ha terminado de exponer las demás, concretamente dice:

Es suficiente lo que se ha dicho de las servidumbres, del usufructo, del uso y de la habitación. De las herencias y las obligaciones, trataremos en sus respectivos lugares. Hemos expuesto sumariamente de qué modos se adquieren las cosas Iure gentium, ahora veamos de qué modos se adquieren por derecho legítimo y civil. ${ }^{19}$

$$
\text { (Inst., 2, 5, 6) }
$$

Es decir, expone lo que no le representa mayor complicación o que no es muy extenso, y deja los temas densos o amplios para ser tratados aparte, con suficiente espacio. De esta suerte, puede constatarse que el tema de la herencia ocupa casi la mitad restante del libro II y la mitad del libro III; y el de las obligaciones, el resto del libro III y prácticamente todo el libro IV.

Acorde con este modo de presentar los temas, existen a lo largo del texto constantes remisiones al Digesto o al Código,

${ }^{19}$ Haec de servitutibus et usu fructu et usu et habitatione dixisse sufficiat. de hereditate autem et de obligationibus suis locis proponemus. exposuimus summatim, quibus modis iure gentium res adquiruntur: modo videamus, quibus modis legitimo et civili iure adquiruntur. 
donde el asunto está más ampliamente tratado. Así, por ejemplo, en Inst., 1, 10, 11, luego que ha expuesto sobre quiénes pueden contraer matrimonio, y con quiénes no - aludiendo a los grados de parentesco-, dice que "hay otras personas que por diversas razones no pueden contraer nupcias, cuyas causas hemos hecho numerar en los libros del Digesto o Pandectas colegidos a partir del antiguo derecho". ${ }^{20}$ Otro ejemplo se halla en el tema de las acciones (Inst., 4, 6, 37), al que remite para ser visto con mayor extensión en los libros del Digesto.

Pero, además de su intención de comenzar por explicar lo sencillo, es notoria también la forma esquemática en que lo hace, siguiendo un orden lógico, característica exigida, por cierto, en la definición de "sistema", como se mencionó al inicio de esta exposición. Esto puede apreciarse en el siguiente pasaje, ubicado justo cuando ha terminado de exponer las divisiones del Derecho y ha definido sus diversas fuentes:

Así, todo el Derecho del que hacemos uso, se refiere ya a las personas, ya a las cosas, ya a las acciones. Pero tratemos primero el de las personas, porque poco se conoce el Derecho si se ignoran las personas por cuya causa ha sido constituido. ${ }^{21}$

(Inst., 1, 2, 12)

Dicho lo cual, en el párrafo siguiente (Inst., 1, 3 pr.), explica la Summa ...divisio de iure personarum.

También expone esquemáticamente una clasificación en 3 , 13, 1 y 2, de mayor a menor, y de general a específico, donde afirma que las obligaciones se reducen a una división principal de dos clases, o son civiles o pretorianas, indicando que sigue luego una división en cuatro especies.

\footnotetext{
${ }^{20}$ Sunt et aliae personae, quae propter diversas rationes nuptias contrahere prohibentur, quas in libris digestorum seu pandectorum ex veteri iure collectarum enumerari permisimus.

${ }^{21}$ Omne autem ius, quo utimur, vel ad personas pertinet vel ad res vel ad actiones. ac prius de personis videamus. nam parum est ius nosse, si personae, quarum causa statum est, ignorentur.
} 
Pero lo que puede considerarse el ejemplo más importante de esquematización en las Instituciones de Justiniano, es la detallada enumeración que ofrece sobre los grados de parentesco (Inst., 3, 6, 9), respecto a la cual comenta lo siguiente:

Pero como la verdad se graba en el ánimo con el testimonio de los ojos más que con el del oído, creímos necesario, después de explicar los grados, presentarlos también inscritos en el presente libro...22

Respecto a los signos de cohesión en las Instituciones, pueden mencionarse los cierres de tema que presenta con frecuencia al final de un apartado, con frases como "es suficiente lo que se ha dicho de tal tema", refiriéndose a un tema en específico. Son cierres que con frecuencia aprovecha para introducir o anunciar, a manera de encabalgamiento, el tema del siguiente título, como lo vemos al iniciar el libro segundo, donde dice: "En el libro anterior expusimos el derecho relativo a las personas; ahora tratemos de las cosas..."23 (2, 1 pr.).

Sin embargo, hay también muchos ejemplos donde se introduce el nuevo tema, no al final del apartado anterior, sino al principio del título correspondiente como, por ejemplo, en 3, 7 pr. "Ahora trataremos de los bienes de los libertos"; ${ }^{24}$ o en 3, 13 pr. "Pasemos ahora a las obligaciones...". ${ }^{25}$

Hay también alusiones a temas no contiguos, a manera de reenvíos, como en este pasaje:

Veamos ahora de qué manera las personas sometidas al derecho de otro se liberan de ese derecho. Y en efecto, ya sabemos cómo

\footnotetext{
22 Sed cum magis veritas oculata fide quam per aures animis hominum infigitur, ideo necessarium duximus post narrationem graduum etiam eos praesenti libro inscribi...

${ }^{23}$ Superiore libro de iure personarum exposuimos: modo videamus de rebus.

${ }^{24}$ Nunc de libertorum bonis videamus.

${ }^{25}$ Nunc transeamus ad obligationes.
} 
son liberados de una potestad los esclavos, porque ya arriba expusimos acerca de las manumisiones... ${ }^{26}$

$$
(1,12 \text { pr.) }
$$

Además de estas conexiones e interconexiones en el discurso de las Instituciones de Justiniano, se encuentran elementos reiterativos que refuerzan o enfatizan su carácter didáctico. Se dan casos como el siguiente: "Retomando todo lo que ya hemos dicho, se advierte que los que se hallan en un mismo grado de cognación, no son siempre de igual modo llamados $<$ a heredar>". ${ }^{27}$

En un terreno más bien de la historia, del devenir de la cultura jurídica reflejada en el texto que nos ocupa, conviene hacer también ciertos señalamientos que forman parte de estas características sistemáticas en las Instituciones. De esta suerte, en el tratamiento de los temas se observa que Justiniano remite con frecuencia a sus antecedentes en la antigüedad clásica, y en ocasiones incluso a las Doce Tablas. En dichos casos suele comenzar por hacer la historia, explicando cómo era tal institución en Roma, quién la emitió o en qué época, para luego, generalmente, concluir con su propuesta, manifestando la causa por la que hace el cambio, o cuál es su intención, así como el modo en que se adecua a las nuevas circunstancias. Muchas de sus resoluciones significaron, por cierto, una política de equidad en varios sentidos, suprimiendo desigualdades sociales, incluyendo medidas más humanitarias para las mujeres, para libertos o para esclavos, y reflejando, asimismo, una tendencia simplificadora pero a la vez incluyente, en términos sociales. Así, son de llamar la atención las leyes que benefi-

\footnotetext{
${ }^{26}$ Videamus nunc, quibus modis ii, qui alieno iuri subiecti sunt, eo iure liberantur. et quidem servi quemadmodum potestate liberantur, ex his intellegere possumus, quae de servis manumittendis superius exposuimus.

${ }^{27}$ 3, 6, 11: Repetitis itaque omnibus quae iam tradidimus apparet non semper eos, qui parem gradum cognationis optinent, pariter vocari...
} 
cian a los esclavos, ${ }^{28}$ a los libertini, ${ }^{29}$ o a la mujer, sobre todo en el contenido de los títulos del libro tercero. Son disposiciones en las que, con frecuencia, lleva un rescripto que había sido emitido para un determinado caso, a una aplicación más general, como puede observarse en Inst., 3, 11, 3 y 4, a propósito de un tipo de sucesión.

\section{La lengua de las Instituciones}

El latín en que está escrito el texto es, en general, sencillo, salvo por la dificultad que representa la terminología jurídica que contiene. ${ }^{30}$ No obstante, para efectos de traducción, es imprescindible respetar dicha terminología, pues de este modo se evita el riesgo de desvirtuar los conceptos técnicos o especializados que transmite un texto de tal naturaleza.

Aunque el texto de las Instituciones está escrito en latín, Justiniano hace frecuentes aclaraciones respecto a cómo "se dice en griego" tal término. Este recurso también lo utilizará de modo muy frecuente en las explicaciones etimológicas, que analizaré en su lugar específico. A propósito de las lenguas, y más allá de los recursos importantes que acabamos de observar para la comprensión del texto, prevalece el sentido práctico de Justiniano en el título acerca de las estipulaciones, en el que dice lo siguiente: "No importa si la estipulación se hace en lengua latina o griega, o en cualquier otra lengua, con tal que los estipulantes la entiendan..."31 (Inst., 3, 15, 1).

\footnotetext{
${ }^{28}$ Por ejemplo, contra la crueldad de los esclavos en 1, 8, 2 y 1, 8, 8 in fine.

${ }^{29}$ Especialmente a los peregrinos dediticios, Inst., 1, 5, 3.

${ }^{30}$ Sobre las características de textos técnicos y especializados como los jurídicos, hay que hacer notar que, dada su especialidad, están dirigidos a un público restringido de iniciados y profesionales, con el objetivo de transmitir, sin elaboración estética en particular, una información científica y técnica.

${ }^{31}$ Utrum autem Latina an Graeca vel qua alia lingua stipulatio concipiatur, nihil interest, scilicet si uterque stipulantium intellectum huius linguae habeat.
} 
Además de los términos técnicos que son reflejo de estructuras de expresión y que muestran un modelo preestablecido o estereotipado de enunciados y, por el contrario, de los modos de expresión transformados, que con frecuencia son reflejo de los cambios en el tratamiento del contenido jurídico respecto del derecho clásico, se introducen usos coloquiales de la lengua. De esta suerte, vemos que Justiniano utiliza expresiones de uso corriente como, por ejemplo, cuando en Inst., 1, 1, 1, aconseja no abrumar al estudiante con demasiada información pues - dice - "una de dos (duorum alterum), o lo convertimos en desertor de sus estudios o ... lo llevaríamos más tarde a aquello que hubiera podido llegar más oportunamente". ${ }^{32}$ Asimismo, como reflejo de la evolución de la lengua latina, aparecen formas menos sintéticas de expresión, como "in meliorem" (Inst., 1, 5, 3) o "in melius" (Inst., 1, 12, 6), una forma que tiende a ser analítica, usada en lugar de un adverbio o de un ablativo, que serían formas más acordes con el latín clásico.

Encontramos también repetición de expresiones, como por ejemplo "omnibus modis" (Inst., 1, 15, 3), usada dos veces en un mismo parágrafo y muy cerca una de la otra; y una presencia reiterada de ciertas partículas como autem, citada decenas de veces sólo en el libro primero. Asimismo la forma item, que aparece 26 veces al inicio de varios párrafos; la palabra tamen (22 veces), e itaque (15 veces). La palabra autem termina siendo una muletilla, pues la mayoría de los usos que tiene en el texto representa un sentido tan sutil, que resulta intraducible. Por su parte, item es usado a veces tan cercano uno de otro, que resulta muy notorio. Esto puede reflejar, o bien una cierta pobreza del lenguaje, o bien que, debido a la tarea selectiva de los pasajes que quedaron fijados en el cuerpo de las Instituciones, los compiladores no pulieron la redacción en su conjunto; pero en todo caso, son signos de una lectura reiterativa.

\footnotetext{
${ }^{32}$ Cf. nota 18 .
} 
A diferencia del resto del Corpus, el texto de las Instituciones tiene una redacción corrida, es decir, no encontramos cortes ni señalamientos de las citas de las fuentes de donde fueron extraídos los conceptos, como sí se hace, por ejemplo, en el Digesto. No obstante lo anterior, presenta una subdivisión por libros, títulos y fragmentos.

En cuanto a las características del discurso, hay que decir que están escritas en un estilo muy directo. En todo momento se habla al interlocutor de la misma manera en que un maestro suele hablar a sus discípulos en el aula, y con un lenguaje sencillo y claro. El discurso se da siempre en primera persona del plural, de un modo expositivo que le habla directamente a un tú: así, por ejemplo, en Inst., 1, 2, 1, cuando acaba de explicar las diferencias entre el ius naturale, el ius gentium y el ius civile, y concluye diciendo que el pueblo romano hace uso en parte de su propio derecho (ius civile) y en parte de un derecho común a todos los hombres (ius gentium), alude a las divisiones del derecho civil, y cierra el parágrafo diciendo: "En sus respectivos lugares expondremos cuáles son cada uno de éstos"; 33 o bien, en el párrafo inicial de Inst., 1, 12, a propósito de los modos mediante los cuales se disuelve la patria potestad, donde, luego de concluir la exposición sobre las adopciones (Inst., 1, 11), cambia de tema y dice: "Veamos ahora de qué modos los que están sometidos a un derecho ajeno son liberados de éste"; ${ }^{34}$ o bien, cuando se dispone a iniciar el tema de las tutelas: "Pasemos ahora a otra división de <el tema de> personas", ${ }^{35}$ etcétera.

\footnotetext{
${ }^{33}$ Quae singula qualia sint, suis locis proponemus.

${ }^{34}$ Videamus nunc, quibus modis ii, qui alieno iuri subiecti sunt, eo iure liberantur.

${ }^{35}$ Transeamus nunc ad aliam divisionem personarum.
} 


\section{El gusto de Justiniano por la etimología}

Un tratamiento especial merece el estudio de la presencia de alusiones etimológicas en el texto justinianeo, característica que, como veremos, contribuye a reforzar su índole didáctica.

En primer lugar, hay que considerar que la explicación etimológica aparece a lo largo de la obra, y la frecuencia con la que se presenta indujo ya a un análisis independiente ${ }^{36}$ con el que están vinculados algunos casos que aquí analizo.

Así pues, el interés etimológico de Justiniano comienza allí donde dice que "el derecho civil es denominado a partir del nombre de cada ciudad". Refiriéndose en primer lugar al derecho griego con la aclaración de que “... aunque si alguien quisiera llamar derecho civil de los atenienses a las leyes de Solón o de Dracón, no errará...", después de explicar la denominación del derecho romano, termina diciendo: “... igualmente llamamos derecho civil de los romanos al derecho del que hace uso el pueblo romano, o Ius Quiritium, del cual hacen uso los Quirites, pues los romanos son nombrados Quirites a partir de Quirino"37 (Inst., 1, 2, 2).

Más adelante encontramos la explicación que ofrece a propósito de los esclavos (servi) tomados a causa de guerra, de los cuales nos dice que se denominan así por el hecho de que los generales que los capturaban, preferían conservarlos (servare) en lugar de matarlos (Inst., 1, 3, 3). El texto relaciona servi con el verbo servare, cuya vinculación de raíz es muy clara, independientemente de que los conservaban vivos para

\footnotetext{
${ }^{36}$ Vid. "La etimología en las Instituciones de Justiniano", Segundo Encuentro Internacional de Retórica, México, UNAM/IIFL, 2003.

${ }^{37}$ Sed ius quidem civile ex unaquaque civitate appellatur, veluti Atheniensium: nam si quis velit Solonis vel Draconis leges apellare ius civile Atheniensium, non erraverit. sic enim et ius, quo populus Romanus utitur, ius civile Romanorum appellamus: vel ius Quiritium, quo Quirites utuntur: Romani enim a Quirino Quirites appellantur.
} 
obtener un precio por ellos, según lo afirma el propio texto justinianeo. Allí mismo, encontramos además la causa por la que estos esclavos se denominan mancipia: "porque son tomados (capiuntur) de entre los enemigos con la mano (manu)". Debemos añadir que el término manus tiene también el sentido de "poder adquirido sobre una persona libre", ${ }^{38}$ de modo que incluso, además del sentido literal de este pasaje, "manu" puede también significar "mediante manus", es decir, mediante la potestad que eventualmente puede tener un pater familias respecto de una persona libre.

Un ejemplo más es el que presenta a propósito de la explicación de tutela (Inst., 1, 13, 2). El autor declara: "Y son tutores los que tienen aquella fuerza y potestad precisamente a partir de la cual tomaron el nombre. Y así se llaman tutores como si fueran tuitores $^{39}$ y defensores, tal como son llamados aeditui $^{40}$ los que protegen los Aedes". ${ }^{41}$ Nótese aquí cómo recurre incluso a dar otra etimología (aeditui) para explicar la primera (tuitores). Así como estos ejemplos, hay varios más en la obra.

En términos generales, las etimologías referidas por Justiniano son muy acertadas, con lo cual demuestra una competencia en el uso de la lengua. Pero más allá del estudio intrínseco de las etimologías, cabe ante todo preguntarse sobre la causa de su utilización en un texto de esta índole. Lo que se puede pensar es que muy probablemente se deba a la elementalidad de la obra, a que es un texto introductorio a una ciencia, y en este sentido, es natural comenzar por definir los términos. El comenzar a dilucidar un concepto mediante

\footnotetext{
${ }^{38}$ Cf. Faustino Gutiérrez Alviz, Diccionario de Derecho Romano, s. v. mancipium.

${ }^{39}$ tuitores ... de tueor, proteger; por tanto, protectores.

${ }^{40}$ De aedes, templos y tueor, proteger; por tanto, protectores de templos.

${ }^{41}$ Tutores autem sunt, qui eam vim ac potestatem habent, ex qua re ipsa nomen ceperunt. Itaque appellantur tutores quasi tuitores atque defensores, sicut aeditui dicuntur qui aedes tuentur.
} 
la explicación del propio término que lo denomina, nos lleva por fuerza a considerar que la palabra se concibe como vía de acceso al conocimiento; y para transmitir conocimiento a través la explicación de la palabra, qué mejor que comenzar por la etimología.

Aunado a este gusto por la explicación etimológica, y relacionado también con el ámbito de la terminología, cabe llamar la atención sobre el siguiente hecho: a lo largo de toda la Edad Media y todavía en épocas más cercanas a nosotros, la cátedra de Instituta se enseñaba a partir de las Instituciones de Justiniano en ediciones que solían ir acompañadas de dos libros del Digesto: el De verborum significatione, "Sobre el significado de las palabras" (D., 50, 16) y el De diversis regulis iuris antiqui, "Sobre las diversas reglas del Derecho antiguo" (D., 50, 17), es decir, precisamente los libros que contenían la terminología jurídica de uso más frecuente con su respectiva explicación. ${ }^{42}$ Ambos títulos son los últimos de todo el Digesto y, al parecer, no tienen relación temática ni con el propio libro 50, al que pertenecen, ni con el resto del Digesto en general. Esta es una vinculación que resulta reveladora, pero sobre todo lógica si consideramos que ésos eran los pasajes del Corpus apropiados para la enseñanza, al igual que las Instituciones. Hay que agregar además que, incluso, los profesores de la cátedra de Digesto a menudo solían comenzar su curso con el último título $(D ., 50,17)$. Seguramente por la utilidad didáctica que les reportaba. ${ }^{43}$

\footnotetext{
${ }^{42}$ Cf. Aurelia Vargas, Instituciones de Justiniano en Nueva España, pp. 43, 50 y 95 .

${ }^{43}$ Archi, a propósito del los géneros literarios institutiones y regulae de la obra del jurista Ulpiano, que califica de "strettamenti affini", declara: "Opere cioé che si propongono l'e sposizione dei principii fondamentali dell' ordinamento giuridico" (Gian Gualberto Archi, "Giustiniano e l'insegnamento del Diritto", en $L$ ' imperatore Giustiniano storia e mito, p. 129).
} 


\section{Conclusiones}

Existe una constante en el discurso de Justiniano. El autor suele plantear un principio o una institución jurídica comenzando por exponer cómo era en el derecho antiguo, con la intención de evidenciar que conoce los antecedentes de la institución en cuestión, para luego, de una manera en general poco modesta, ${ }^{44}$ justificar las adaptaciones o reformas que introduce, reuniéndolas muchas veces en una solución general. Este hecho ha provocado que, por la recopilación de las fuentes antiguas, unos lo consideran "clasicista"; pero otros "vulgarizador", por los cambios que introduce.

Vulgarizador o no, lo cierto es que, mediante su compilación, Justiniano brindó respuesta a los retos que su momento histórico le exigía, pues estaba obligado a adecuarse a sus circunstancias; y en todo caso, es una tendencia vulgarizadora en el campo del Derecho que también repercute en la lengua. Pero hablando en términos linguísticos e históricos, las Instituciones son un reflejo del modo de hablar de su época. Esta polémica nos recuerda ciertas ideas muy difundidas, y que todavía hoy tiene sus seguidores, respecto al cambio lingüístico - concretamente el cambio del latín respecto de las lenguas neolatinas - , en el sentido de considerar como corrupciones o vulgarizaciones los cambios que en realidad son evoluciones naturales de una entidad cultural, en este caso la lengua. Con las instituciones jurídicas sucede algo semejante, y en este caso particular, Justiniano no está haciendo otra cosa que aprovechar lo que la tradición le ha legado, y recibirla para aplicarla, a través de su legislación, en su momento histórico.

\footnotetext{
${ }^{44}$ Por ejemplo, en Inst., 1, 5, 3 ... en otra constitución que brilla... cuando habla de que suprimió todas las diferencias entre libertinos, dotando por igual de la ciudadanía romana a todos sin distinción. Y efectivamente, Justiniano abolió el estado de los dediticios. Vid. Jesús García Garrido, Diccionario de Jurisprudencia Romana, s. v. dediticii.
} 
Es pertinente aquí la distinción que introduce Braudel ${ }^{45}$ sobre los fenómenos históricos, según la cual, en el devenir de las civilizaciones existen fenómenos de corta, mediana y larga duración. Se trata de una concepción interesante en la que los dos primeros tipos de fenómenos sólo se explican a la luz del de larga duración, lo cual vendría siendo aquello que permanece como una especie de sustrato, en la medida que siguen existiendo en la realidad las condiciones que lo mantienen; sustrato que a la vez funciona como un elemento común y cotidiano, tan cotidiano, que se hace imperceptible, pero que se manifiesta a la menor provocación. El objetivo de Justiniano se logró al ofrecer un texto docente y legislativo del todo accesible, suficientemente explicado, y recuperador de una importante tradición.

Hemos analizado algunos aspectos que consideramos sistemáticos en las Instituciones de Justiniano, tanto en su composición formal como en su estilo y en sus contenidos jurídicos. El hecho de que el discurso sea corrido, ausente de citas formales pero con frecuentes remisiones a otras secciones del hoy llamado Corpus Iuris Civilis, ha hecho que se le considere - en una clara correspondencia con su extensión y su nivelcomo un prontuario o un resumen introductorio a todo el Corpus. Hemos visto que es una obra de índole esencialmente didáctica, tanto por su exposición en lenguaje claro y sencillo, como por sus reiteraciones y sus explicaciones etimológicas; asimismo, hemos visto que es una obra de utilidad práctica, no sólo por su utilización en la propia docencia, sino por su aplicación como ley vigente. Justiniano brindó sus Instituciones en un tono que incluso podríamos calificar de "amigable", para acompañar en sus esfuerzos, allanando un camino que él mismo había recorrido, "a la juventud estudiosa".

\footnotetext{
${ }^{45}$ Fernand Braudel, "La larga duración”, en La historia y las ciencias sociales, passim.
} 


\section{BIBLIOGRAFÍA}

\section{Fuentes}

"Digesta Iustiniani”, en Corpus Iuris Civilis, Theodor Mommsen (ed.), Dublín, Weidmannos, 1963, vol. 1.

El Digesto de Justiniano, trad. Álvaro d'Ors et al., Pamplona, Editorial Aranzadi, 1968-1975, 3 vols.

GAYo, Instituciones, Fco. Hernández Tejero (coord.), Madrid, Editorial Civitas, 1985.

"Institutiones Iustiniani”, en Corpus Iuris Civilis, Theodor Mommsen (ed.), Dublín, Weidmannos, 1963, vol. 1.

Ortolán, M., Instituciones de Justiniano, trad. esp. Francisco Pérez de Anaya y Melquiades Pérez Rivas, Buenos Aires, Heliasta, 1976.

\section{Estudios}

ArchI, Gian Gualberto, "Giustiniano e l'insegnamento del Diritto", en L' imperatore Giustiniano storia e mito, Milán, Giuffré, 1978.

BRAUdEL, Fernand, "La larga duración", en La historia y las ciencias sociales, Madrid, Alianza, 1986 (7a. reimp.).

D’Ors, Álvaro, Derecho Privado Romano, Pamplona, Ediciones Universidad de Navarra, 1991.

KÁser, Max, Derecho Romano Privado, Madrid, REUS, 1968.

Padilla Sahagún, Gumesindo, Derecho Romano, México, Mc Graw-Hill, 2008 (4a. ed.).

Vargas Valencia, Aurelia, Las Instituciones de Justiniano en la Nueva España, México, UNAM/Instituto de Investigaciones Filológicas, 2001.

\section{Diccionarios y léxicos}

Berger, Adolf, Encyclopedic Dictionary of Roman Law, Philadelphia, American Philosophical Society, 1968.

ForCELlini, Aegidius, Lexicon totius latinitatis, Padua, U. Gregoriana, 1965.

García Garrido, Jesús, Diccionario de Jurisprudencia Romana, Madrid, Dykinson, 1982. 
Gutiérrez Alviz, Faustino, Diccionario de Derecho Romano, Madrid, REUS, 1982.

Real Academia Española, Diccionario de la lengua española, Madrid, Espasa Calpe, 1992. 